\title{
Mixed convolved action variational principles in heat diffusion
}

\author{
G.F. Dargush*, G. Apostolakis ${ }^{\dagger}$, B.T. Darrall, J. Kim ${ }^{\ddagger}$ \\ Department of Mechanical \& Aerospace Engineering, University at Buffalo, State University of New York, Buffalo, \\ NY 14260
}

\begin{abstract}
A true variational formulation is developed for dissipative processes based upon the concept of mixed convolved action. Here the focus is on continuum problems associated with heat diffusion, as well as related second sound phenomena. The convolved action can overcome the shortcomings of typical action principles, such as Hamilton's principle, to address dissipative processes without the need for separate dissipation functionals and ad hoc variational operations. In addition, the mixed convolved action is compatible with the initial and boundary conditions of a well-posed heat problem. In fact, the stationarity of the mixed convolved action is shown to provide the governing partial differential equations, the initial conditions and the boundary conditions as its Euler-Lagrange equations. Thus, the mixed convolved action encapsulates the entire description of the initial/boundary value heat problem. In addition to the theoretical significance, this new formulation can establish the basis for effective numerical methods, for example, involving finite element representations over both space and time. One particular two-dimensional formulation is developed here and then applied to two example problems to illustrate the viability of the proposed approach.
\end{abstract}

Keywords: Heat diffusion; Variational methods; Hamilton's principle; Euler-Lagrange equations; Time-space finite element methods; Second sound

\footnotetext{
${ }^{*}$ Corresponding author. Tel.: 17166452315.

E-mail addresses: gdargush@buffalo.edu (G.F. Dargush), ga59@buffalo.edu (G. Apostolakis), bdarrall@buffalo.edu (B.T. Darrall),jk295@buffalo.edu (J. Kim)

${ }^{\dagger}$ Present address: Energo Engineering, KBR, 1300 W. Sam Houston Pkwy. S., Suite 100, Houston, TX 77042 USA

‡ Present address: Korea Institute of Disaster \& Safety, 501 ho, Bangjaecenter Bldg., 11, Gyeongin-ro 114ga-gil, Yeongdeungpo-gu, Seoul, Korea
} 


\section{Introduction}

A long-standing problem in theoretical mechanics has been to develop a variational principle for dissipative dynamical systems having the same degree of elegance as is present for conservative systems via Hamilton's principle $[1,2]$. There have been many different approaches advanced over the years. For example, extensions have been proposed through the introduction of a Rayleigh dissipation function [3-6], which was developed extensively for heat transfer by Biot [7, 8], or through the use of mirrored systems $[9,10]$. The former requires acceptance of mathematical anomalies in performing the variations, while the latter necessitates the creation of a fictitious counterpart to the real, physical system of interest. No doubt these approaches can sometimes prove useful, although neither offers a truly satisfactory base for the study of dissipative systems. Consequently, the study of such systems has been regarded as inaccessible by variational methods and other less physically based solution methods have been adopted. The situation can be viewed alternatively as a severe limitation of the classical variational approaches to address many physical problems of interest. Other attempts to include dissipation within a variational approach include generalized bracket formalisms [11-16], complex valued fields [17-19] and fractional derivatives

[20-22]. Recent work on the GENERIC framework [23, 24] has focused on identifying an underlying geometric structure for multiscale dissipative processes that combines the elements of both dynamics and thermodynamics. While this represents significant progress, none of the above formulations in [11-24] has the simplicity of the original approach of Hamilton for conservative systems in which a single functional contains all of the governing physics. In addition, the fractional derivative work [20-22] cannot accommodate classically damped systems, calling into question the validity of those formulations. Closer examination reveals further issues with Hamilton's principle, even for the fully conservative case. More specifically, the temporal end point conditions imposed on the variations are not consistent with the specified initial conditions of the dynamical problem [25-29].

In recent work, all of these shortcomings have been resolved by introducing mathematically consistent variational principles based upon the notion of mixed convolved action [28, 30, 31]. The first two papers deal with spatially discrete single degree-of-freedom dynamic systems [28, 30], while the most recent work addresses elasto- and viscoelasto-dynamic response of solid continua [31]. The mixed convolved action framework extends ideas of Gurtin [25, 32, 33], Tonti 
[26, 27, 34], and Oden and Reddy [35, 36] by using mixed impulsive variables, fractional derivatives and the convolution of convolutions. Most importantly, this framework leads to stationary variational principles that recover all of the governing differential equations, boundary conditions and initial conditions, as the corresponding Euler-Lagrange equations.

The remainder of the paper is organized as follows. In Section 2, we provide a review of the principle of mixed convolved action for discrete dynamical systems. This starts with the classical conservative spring-mass system, where the fundamental ideas can be easily demonstrated. Then, a linear viscous damper is added in series with the spring as a prototype for development of dissipative variational formulations. This leads by analogy to the formulation in Section 3 of a mixed convolved action for the continuum heat diffusion problem. This is extended in Section 4 to thermal problems in which finite propagation speeds must be maintained. Interestingly, within the mixed convolved action framework, such problems, which are finding increased importance in modern technology, can be readily addressed. This new theoretical framework also provides an interesting base for computational methods. In Section 5, the mixed convolved action is discretized in both space and time to provide a pure variational finite element method for heat diffusion. Several computational examples are considered in Section 6 to validate these novel variational methods for non-conservative systems. Finally, Section 7 presents some general conclusions.

\section{Variational principles for discrete dynamical systems with dissipation}

Let us begin with perhaps the simplest conservative dynamical system involving the unidirectional motion of a mass $m$ connected to a support through a linear spring with constant stiffness $k$. Hamilton's principle for this system is based upon the definition of the following Lagrangian:

$$
L(u, \dot{u} ; t)=\frac{1}{2} m \dot{u}^{2}-\frac{1}{2} k u^{2}+\bar{f} u
$$

where $u(t)$ is the displacement of the mass from its equilibrium position with the spring

unstretched and $\bar{f}(t)$ is an applied force, both as functions of time $t$. As usual, each superposed dot represents a time derivative.

The action functional $I_{H}$ for Hamilton's principle over the fixed time interval from 0 to $t$ is written 


$$
I_{H}(u, \dot{u} ; t)=\int_{0}^{t} L(u, \dot{u} ; \tau) d \tau
$$

For stationary action, the first variation of Eq. (2) must be zero, which after applying integration by parts, becomes

$$
\delta I_{H}=-\int_{0}^{t}[m \ddot{u}+k u-\bar{f}] \delta u d \tau+[m \dot{u} \delta u]_{0}^{t}=0
$$

Note that in arriving at Eq. (3), the applied force $\bar{f}$ is assumed known and, therefore, not subject to variation.

Using the approach of Hamilton $[1,2]$ to recover the governing equation of motion, we also must invoke the condition of zero variation of displacement at the beginning $\delta u(0)$ and end $\delta u(t)$ of the time interval to nullify the last bracketed terms in Eq. (3). With those additional constraints, Eq. (3) reduces to

$$
\delta I_{H}=-\int_{0}^{t}[m \ddot{u}+k u-\bar{f}] \delta u d \tau=0
$$

and after permitting arbitrary variations $\delta u$ between the endpoints, we have the equation of motion for forced vibration

$$
m \ddot{u}+k u=\bar{f}
$$

at each instant of time $\tau$ with $0<\tau<t$. Thus, Eq. (5) is the Euler-Lagrange equation associated with the stationarity of the action $I_{H}$. This result also can be obtained by invoking the Lagrange formalism and simply writing

$$
\frac{d}{d t} \frac{\partial L}{\partial \dot{u}}-\frac{\partial L}{\partial u}=0
$$

Either way, nothing can be said concerning the initial conditions, which must be enforced separately, outside of Hamilton's principle. More disturbing are the impositions on the variations of the displacement, which are inconsistent with the specified initial conditions. How can one impose zero variation at the end of the time interval, when the displacement at that instant is precisely the unknown quantity of most interest?

Gurtin $[25,32,33]$ and Tonti $[26,27,34]$ were the first to recognize that the inner products appearing in Eq. (2) must be replaced by convolutions. However, even this is not enough to provide a variational principle that recovers not only the governing differential equation of motion, 
but also the initial conditions, as its Euler-Lagrange equations. Based upon the work in Reference [28], we write the mixed convolved action for the conservative spring-mass system in the following form:

$$
I_{C}(u, \breve{u}, \dot{u}, J, \breve{J}, \dot{J} ; t)=\frac{1}{2} m \dot{u} * \dot{u}-\frac{1}{2} a \dot{J} * \dot{J}+\breve{u} * \breve{J}-\breve{u} * \breve{j}
$$

where again $u$ represents the displacement of the mass from the equilibrium position, while $J$ is the impulse of the elastic force $F$ in the linear spring, such that

$$
J(t)=\int_{0}^{t} F(\tau) d \tau
$$

Meanwhile, $a=1 / k$ is the flexibility of the spring and the superposed breve denotes a left Riemann-Liouville semi-derivative. Thus, for example,

$$
\breve{u}(t)=\left(\mathscr{D}_{0^{+}}^{1 / 2} u\right)(t) \equiv+\frac{1}{\Gamma(1 / 2)} \frac{d}{d t} \int_{0}^{t} \frac{u(\xi)}{(t-\xi)^{1 / 2}} d \xi
$$

Details on fractional calculus can be found, for example, in Oldham and Spanier [37], Ross [38] and Samko et al. [39]. Fractional integration by parts formulas, which are needed in the following development, were first formulated by Love and Young [40]. Close comparison of Eq. (62) of Reference [28] with Eq. (7) above reveals a slightly more graceful treatment of the applied force term in the present work, which removes the need for an explicit reference to an initial impulse.

Taking variations of the mixed convolved action of Eq. (7) yields the following weak form:

$$
\delta I_{C}=\delta \dot{u} * m \dot{u}-\delta \dot{J} * a \dot{J}+\delta \breve{J} * \breve{u}+\delta \breve{u} * \breve{J}-\delta \breve{u} * \breve{j}
$$

After applying classical integration-by-parts to the first and second terms and fractional integration-by-parts to the third, fourth and fifth terms on the right-hand side of Eq. (10), one may collect terms and write for stationary mixed convolved action:

$$
\begin{aligned}
& \delta I_{C}=\delta u *[m \ddot{u}+\dot{J}-\bar{f}]+\delta J *[-a \ddot{J}+\dot{u}] \\
& +\delta u(t)[m \dot{u}(0)+J(0)-\bar{j}(0)]+\delta u(0)[m \dot{u}(t)] \\
& +\delta J(t)[-a \dot{J}(0)+u(0)]-\delta J(0)[-a \dot{J}(t)]=0
\end{aligned}
$$

Several results on fractional integration by parts formulas used in this step can be found in References [28, 30].

For arbitrary variations $\delta u$ and $\delta J$, with the initial constraints

$$
\delta u(0)=0, \quad \delta J(0)=0
$$


one may recover from Eq. (11), the governing differential equations

$$
\begin{array}{r}
m \ddot{u}+\dot{J}=\bar{f} \\
-a \ddot{J}+\dot{u}=0
\end{array}
$$

and the initial conditions

$$
\begin{aligned}
& m \dot{u}(0)+J(0)=\bar{j}(0) \\
& -a \dot{J}(0)+u(0)=0
\end{aligned}
$$

Thus, for the spring-mass system, both the governing differential equations and initial conditions in mixed form are obtained as the Euler-Lagrange equations associated with $I_{C}$. This is clearly an advantage over the corresponding formulation based upon Hamilton's principle, which is inconsistent with the specified initial conditions. We have worked here with the formulation in mixed variables. However, by integrating Eq. (13b) over time from 0 to $t$, imposing initial condition Eq. (14b) and then substituting into Eq. (13a), the usual equation of motion in displacement form can be obtained.

As a further advantage of the mixed convolved action formulation, Eq. (7) can be readily extended to incorporate linear dissipative elements. For a linear dashpot in series with the spring, one may write for the resulting Maxwell model

$$
I_{C_{M}}(u, \breve{u}, \dot{u}, J, \breve{J}, \dot{J} ; t)=\frac{1}{2} m \dot{u} * \dot{u}-\frac{1}{2} a \dot{J} * \dot{J}+\breve{u} * \breve{J}-\frac{1}{2} d \breve{J} * \breve{J}-\breve{u} * \breve{j}
$$

with $d$ as a dashpot coefficient. Following the same steps as for the conservative spring-mass system, stationary action becomes

$$
\delta I_{C_{M}}=\delta \dot{u} * m \dot{u}-\delta \dot{J} * a \dot{J}+\delta \breve{J} * \breve{u}+\delta \breve{u} *-\delta \breve{J} * d \breve{J}-\delta \breve{u} * \breve{j}=0
$$

After classical and fractional integration-by-parts, this can be rewritten by collecting terms according to the variations as

$$
\begin{aligned}
& \delta I_{C_{M}}=\delta u *[m \ddot{u}+\dot{J}-\bar{f}]+\delta J *[-a \ddot{J}-d \dot{J}+\dot{u}] \\
& \quad+\delta u(t)[m \dot{u}(0)+J(0)-\bar{j}(0)]+\delta u(0)[m \dot{u}(t)] \\
& \quad+\delta J(t)[-a \dot{J}(0)-d J(0)+u(0)]-\delta J(0)[-a \dot{J}(t)]=0
\end{aligned}
$$

Constraining the variations to be consistent with the initial conditions once again requires Eq. (12a,b) to hold. Finally, for otherwise arbitrary variations, Eq. (17) necessitates

$$
m \ddot{u}+\dot{J}=\bar{f}
$$




$$
-a \ddot{J}-d \dot{J}+\dot{u}=0
$$

along with the initial conditions

$$
\begin{gathered}
m \dot{u}(0)+J(0)=\bar{j}(0) \\
-a \dot{J}(0)-d J(0)+u(0)=0
\end{gathered}
$$

which are precisely the relations for this spring-dashpot-mass system. For this case with the spring and dashpot in series, it is not possible to combine Eqs. (18a) and (18b) into a single governing differential equation of motion in terms of displacement. On the hand, it is possible to write a single flexibility differential equation in terms of impulse $J$.

As demonstrated in this section, conservative and non-conservative systems can be accommodated within the same variational framework of the mixed convolved action and, furthermore, both the governing differential equations and initial conditions are produced as the Euler-Lagrange equations of this action. With the success of this approach, an obvious question is whether this framework can be extended to continua. As a prototype, problems of heat diffusion are addressed in the following section.

\section{Variational principles for continuum heat diffusion}

Based upon the success in developing stationary principles for discrete conservative and dissipative systems within a common framework, we next formulate for the first time analogous variational principles for heat diffusion in a continuum. We should emphasize that these principles recover all of the governing partial differential equations, initial conditions and boundary conditions of the well-posed heat diffusion problem. Two cases are considered. First, classical heat conduction based upon Fourier's law of heat conduction is addressed in this section and then

afterwards in Section 4 the formulation is extended to a generalized model that restricts the propagation of the thermal effects to finite speed.

As in the discrete mixed convolved actions presented in Section 2 and recent mixed Lagrangian formulations [41-45], the thermal field will be described by impulsive variables. Thus, we choose for the primary variables $\theta$, which represents the impulse of temperature $T$, and the heat vector $H_{i}$, which can be considered as the impulse of the heat flux $q_{i}$. In mathematical terms, we may write these variables as 


$$
\begin{gathered}
\theta(t)=\int_{0}^{t} T(t) d t \\
H_{i}(t)=\int_{0}^{t} q_{i}(t) d t
\end{gathered}
$$

over the time interval from 0 to $t$ or in the following rate form

$$
\begin{gathered}
\dot{\theta}=T \\
\dot{H}_{i}=q_{i}
\end{gathered}
$$

Fig. 1 provides a typical problem definition for heat diffusion in two dimensions. In analogy with the discrete action from Section 2 and the inner product thermoelastic formulations from References [43-45], we may write the following mixed convolved action for unsteady Fourier heat conduction:

$$
\begin{aligned}
I_{C_{F}} & =\int_{\Omega}\left[\frac{1}{2} \dot{\theta} * \frac{\rho_{o} C_{\epsilon}}{T_{o}} \dot{\theta}-\frac{1}{2} \breve{H}_{i} * d_{i j} \frac{1}{T_{o}} \breve{H}_{j}\right] d \Omega \\
& -\int_{\Omega}\left[\frac{1}{2}\left(\breve{H}_{i} * \frac{1}{T_{o}} B_{i} \breve{\theta}-\breve{\theta} * \frac{1}{T_{o}} B_{i} \breve{H}_{i}\right)\right] d \Omega-\int_{\Omega}\left[\breve{\theta} * \frac{1}{T_{o}} \breve{\bar{\Psi}}\right] d \Omega \\
& +\int_{\Gamma_{q}} \frac{1}{2}\left[\breve{\theta} * \frac{1}{T_{o}} \breve{Q}\right] d \Gamma-\int_{\Gamma_{T}} \frac{1}{2}\left[\frac{1}{T_{o}} \breve{Q} * \breve{\theta}\right] d \Gamma
\end{aligned}
$$

where $T_{o}$ represents the initial absolute temperature at the free stress state, while $T$ then becomes the temperature change from that state. Additionally, $\rho_{o}$ is the mass density, $c_{\epsilon}$ is the specific heat coefficient at constant strain and $\bar{\psi}$ is a specified body heat source rate per unit volume, with $\bar{\Psi}$ as the impulse of that source. The symbol $B_{i}$ in Eq. (22) represents the gradient operator. Furthermore, with reference to Fig. 1, $\bar{\theta}$ represents the impulse of the enforced boundary temperature $\bar{T}$ on the surface $\Gamma_{T}$, while $\bar{Q}$ denotes the impulse of $\bar{q}$, which defines the specified normal heat fluxes on the surface $\Gamma_{q}$. On the remaining portions of the surface, the default boundary condition is $\bar{q}=0$ representing the insulated condition. Therefore, this also is part of $\Gamma_{q}$. Meanwhile, on $\Gamma_{T}, Q(t)=H_{i}(t) n_{i}=\int_{0}^{t} q_{i}(t) n_{i} d t$ with $n_{i}$ as the outer unit normal to the 
surface. Lastly, the constitutive tensor $d_{i j}$ appearing in Eq. (22) represents the inverse of the conductivity $k_{i j}$.

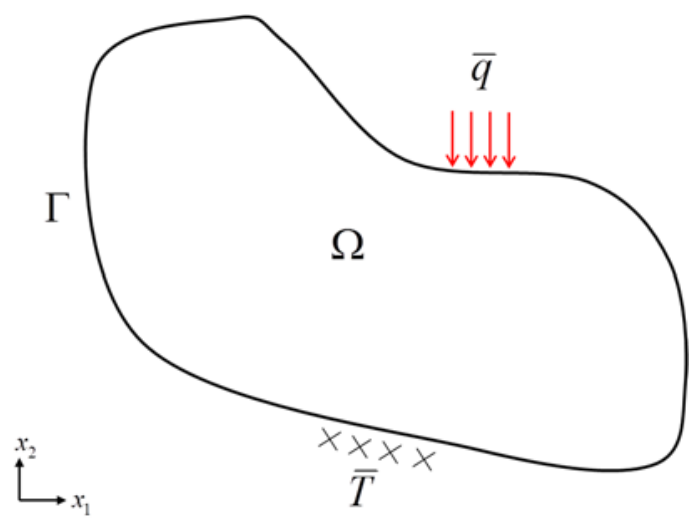

Fig. 1. Heat diffusion problem definition.

Notice that each term in Eq. (22) contains the factor $T_{o}^{-1}$. This is included so that $I_{C_{F}}$ is a true action (i.e., energy integrated over time). While this is not critical for the pure thermal diffusion problem, the formulation proposed in Eq. (22) extends more naturally to a range of multiphysics problems, such as thermoelasticity.

With all of that in mind, the first variation of the mixed convolved action becomes

$$
\begin{aligned}
\delta I_{C_{F}} & =\int_{\Omega}\left[\delta \dot{\theta} * \frac{\rho_{o} C_{\epsilon}}{T_{o}} \dot{\theta}-\delta \breve{H}_{i} * d_{i j} \frac{1}{T_{o}} \breve{H}_{j}\right] d \Omega \\
& -\int_{\Omega}\left[\frac{1}{2}\left(\delta \breve{H}_{i} * \frac{1}{T_{o}} B_{i} \breve{\theta}-\delta \breve{\theta} * \frac{1}{T_{o}} B_{i} \breve{H}_{i}+\frac{1}{T_{o}} B_{i} \delta \breve{\theta} * \breve{H}_{i}-\frac{1}{T_{o}} B_{i} \delta \breve{H}_{i} * \breve{\theta}\right)\right] d \Omega \\
& -\int_{\Omega}\left[\delta \breve{\theta} * \frac{1}{T_{o}} \breve{\Psi}\right] d \Omega+\int_{\Gamma_{q}} \frac{1}{2 T_{o}}[\delta \breve{\theta} * \breve{Q}] d \Gamma-\int_{\Gamma_{T}} \frac{1}{2 T_{o}}[\delta \breve{Q} * \breve{\theta}] d \Gamma
\end{aligned}
$$

After performing the required temporal classical and fractional integration-by-parts operations, as in Section 2, along with spatial integration-by-parts to remove all derivatives from the variations, the stationarity of the mixed convolved action may be written 


$$
\begin{aligned}
\delta I_{C_{F}} & =\int_{\Omega} \delta \theta *\left[\frac{\rho_{o} c_{\varepsilon}}{T_{o}} \ddot{\theta}+\frac{1}{T_{o}} B_{i} \dot{H}_{i}-\frac{1}{T_{o}} \bar{\psi}\right] d \Omega \\
& +\int_{\Omega} \delta H_{i} *\left[-d_{i j} \frac{1}{T_{o}} \dot{H}_{j}-\frac{1}{T_{o}} B_{i} \dot{\theta}\right] d \Omega \\
& +\int_{\Omega} \delta \theta(t)\left[\frac{\rho_{o} c_{\varepsilon}}{T_{o}} \dot{\theta}(0)+\frac{1}{T_{o}} B_{i} H_{i}(0)-\frac{1}{T_{o}} \bar{\Psi}(0)\right] d \Omega \\
& +\int_{\Omega} \delta \theta(0)\left[\frac{\rho_{o} C_{\varepsilon}}{T_{o}} \dot{\theta}(t)\right] d \Omega \\
& +\int_{\Omega} \delta H_{i}(t)\left[-d_{i j} \frac{1}{T_{o}} H_{j}(0)-\frac{1}{T_{o}} B_{i} \theta(0)\right] d \Omega \\
& -\int_{\Gamma_{q}} \frac{1}{2 T_{o}}[\delta \theta * q-\delta \theta * \bar{q}] d \Gamma-\int_{\Gamma_{q}} \frac{1}{2 T_{o}}[\delta \theta(t) Q(0)-\delta \theta(t) \bar{Q}(0)] d \Gamma \\
& -\int_{\Gamma_{T}} \frac{1}{2 T_{o}}[\delta \theta * q] d \Gamma-\int_{\Gamma_{T}} \frac{1}{2 T_{o}}[\delta \theta(t) Q(0)] d \Gamma \\
& +\int_{\Gamma_{T}} \frac{1}{2 T_{o}}[\delta Q * T-\delta Q * \bar{T}] d \Gamma+\int_{\Gamma_{T}} \frac{1}{2 T_{o}}[\delta Q(t) \theta(0)-\delta Q(t) \bar{\theta}(0)] d \Gamma \\
& +\int_{\Gamma_{q}} \frac{1}{2 T_{o}}[\delta Q * T] d \Gamma+\int_{\Gamma_{q}} \frac{1}{2 T_{o}}[\delta Q(t) \theta(0)] d \Gamma=0
\end{aligned}
$$

From Eq. (24) for arbitrary variations, we have as the Euler-Lagrange equations:

$$
\begin{array}{rlr}
\frac{\rho_{o} c_{\varepsilon}}{T_{o}} \ddot{\theta}+\frac{1}{T_{o}} B_{i} \dot{H}_{i}=\frac{1}{T_{o}} \bar{\psi} & x \in \Omega, \tau \in(0, t) \\
-d_{i j} \frac{1}{T_{o}} \dot{H}_{j}-\frac{1}{T_{o}} B_{i} \dot{\theta}=0 & x \in \Omega, \tau \in(0, t) \\
\frac{\rho_{o} c_{\varepsilon}}{T_{o}} \dot{\theta}(0)+\frac{1}{T_{o}} B_{i} H_{i}(0)=\frac{1}{T_{o}} \bar{\Psi}(0) & x \in \Omega \\
-d_{i j} \frac{1}{T_{o}} H_{j}(0)-\frac{1}{T_{o}} B_{i} \theta(0)=0 & x \in \Omega \\
Q(0)=\bar{Q}(0) & x \in \Gamma_{q} \\
\theta(0)=\bar{\theta}(0) & x \in \Gamma_{T} \\
q=\bar{q} & x \in \Gamma_{q}, \tau \in(0, t) \\
T=\bar{T} & x \in \Gamma_{T}, \tau \in(0, t)
\end{array}
$$

with the variations defined, such that

$$
\delta Q=0 \quad x \in \Gamma_{q}, \tau \in(0, t)
$$




$\begin{array}{cc}\delta \theta=0 & x \in \Gamma_{T}, \tau \in(0, t) \\ \delta \theta(0)=0 & x \in \Omega \\ \delta H_{i}(0)=0 & x \in \Omega \\ \delta Q(t)=0 & x \in \Gamma_{q} \\ \delta \theta(t)=0 & x \in \Gamma_{T}\end{array}$

Consequently, we have established a Principle of Stationary Mixed Convolved Action for a Linear Thermal Continuum undergoing classical heat diffusion. This may be stated as follows: Of all the possible trajectories $\left\{\theta(\tau), H_{i}(\tau)\right\}$ of the system during the time interval $(0, t)$, the one that renders the action $I_{C_{F}}$ in Eq. (22) stationary, corresponds to the solution of the initial/boundary value problem of heat diffusion. Thus, the stationary trajectory satisfies the energy balance law Eq. (25a), along with the Fourier law of heat conduction Eq. (25b) in the domain $\Omega$ over the entire time interval. In addition, the initial conditions defined by Eqs. (26a,b) in $\Omega$ and Eqs. (27a,b) on the appropriate portions of the bounding surface are satisfied, while the heat flux Eq. (28a) and temperature Eq. (28b) boundary conditions are satisfied throughout the time interval. Furthermore, the possible trajectories under consideration during the variational process are constrained precisely by their need to satisfy the specified initial and boundary conditions of the problem in the form of Eqs. (29a,b), Eqs. (30a,b) and Eqs. (31a,b).

The usual temperature-based partial differential heat equation can be recovered via the following steps. First, premultiply Eq. (25b) by the symmetric conductivity tensor $k_{i k}$, which produces

$$
-k_{i k} d_{i j} \frac{1}{T_{o}} \dot{H}_{j}-k_{i k} \frac{1}{T_{o}} B_{i} \dot{\theta}=0
$$

or after cancelling terms

$$
\dot{H}_{k}=-k_{i k} B_{i} \dot{\theta}
$$

Then, by substituting Eq. (33) into Eq. (25a), one obtains

$$
\frac{\rho_{o} c_{\varepsilon}}{T_{o}} \ddot{\theta}=\frac{1}{T_{o}} B_{i} k_{i j} B_{j} \dot{\theta}+\frac{1}{T_{o}} \bar{\psi}
$$


which after multiplying through by $T_{o}$ provides the familiar form of the heat equation for anisotropic media.

To summarize, we are now able to define a single real scalar functional, within the mixed convolved action framework based upon convolution and fractional derivatives, which encapsulates the governing differential equations for classical heat diffusion, along with the initial and boundary conditions. This represents the first true variational formulation for a dissipative continuum defined by classical constitutive relations.

\section{Variational principles for continuum heat diffusion with second sound}

With modern technology, there sometimes comes the need to address the actual finite speed of propagation of thermal disturbances and the wave nature of the response. Here we consider one such formulation that involves second sound. Within the mixed convolved action framework, one only needs to modify slightly the specification of Eq. (22) by adding a conservative element, which is associated with a generalized Fourier law of heat conduction. In particular, let

$$
\begin{aligned}
I_{C_{G}} & =\int_{\Omega}[\frac{1}{2} \dot{\theta} * \frac{\rho_{o} C_{\epsilon}}{T_{o}} \underbrace{-\frac{1}{2} \dot{H}_{i} * d_{i j} \frac{\tau_{o}}{T_{o}} \dot{H}_{j}}_{\text {second sound }}-\frac{1}{2} \breve{H}_{i} * d_{i j} \frac{1}{T_{o}} \breve{H}_{j}] d \Omega \\
& -\int_{\Omega}\left[\frac{1}{2}\left(\breve{H}_{i} * \frac{1}{T_{o}} B_{i} \breve{\theta}-\breve{\theta} * \frac{1}{T_{o}} B_{i} \breve{H}_{i}\right)\right] d \Omega-\int_{\Omega}\left[\breve{\theta} * \frac{1}{T_{o}} \breve{\bar{\Psi}}\right] d \Omega \\
& +\int_{\Gamma_{q}} \frac{1}{2 T_{o}}[\breve{\theta} * \breve{Q}] d \Gamma-\int_{\Gamma_{T}} \frac{1}{2 T_{o}}[\breve{Q} * \breve{\theta}] d \Gamma
\end{aligned}
$$

where everything is defined as in Section 3, except for the term labelled as second sound that involves $\tau_{o}$, which represents a relaxation time in the generalized Fourier's law of heat conduction. Taking variations and revisiting all of the steps from Section 3, ultimately provides the following for stationary mixed convolved action: 


$$
\begin{aligned}
& \delta I_{C_{G}}=\int_{\Omega} \delta \theta *\left[\frac{\rho_{o} c_{\varepsilon}}{T_{o}} \ddot{\theta}+\frac{1}{T_{o}} B_{i} \dot{H}_{i}-\frac{1}{T_{o}} \bar{\psi}\right] d \Omega \\
& +\int_{\Omega} \delta H_{i} *[\underbrace{-d_{i j} \tau_{o} \frac{1}{T_{o}} \ddot{H}_{j}}_{\text {second sound }}-d_{i j} \frac{1}{T_{o}} \dot{H}_{j}-\frac{1}{T_{o}} B_{i} \dot{\theta}] d \Omega \\
& +\int_{\Omega} \delta \theta(t)\left[\frac{\rho_{o} c_{\varepsilon}}{T_{o}} \dot{\theta}(0)+\frac{1}{T_{o}} B_{i} H_{i}(0)-\frac{1}{T_{o}} \bar{\Psi}(0)\right] d \Omega \\
& +\int_{\Omega} \delta \theta(0)\left[\frac{\rho_{o} c_{\varepsilon}}{T_{o}} \dot{\theta}(t)\right] d \Omega \\
& +\int_{\Omega} \delta H_{i}(t)[\underbrace{-d_{i j} \tau_{o} \frac{1}{T_{o}} \dot{H}_{j}(0)}_{\text {second sound }}-d_{i j} \frac{1}{T_{o}} H_{j}(0)-\frac{1}{T_{o}} B_{i} \theta(0)] d \Omega \\
& +\int_{\Omega} \delta H_{i}(0)[\underbrace{-d_{i j} \tau_{o} \frac{1}{T_{o}} \dot{H}_{j}(t)}_{\text {second sound }}] d \Omega \\
& -\int_{\Gamma_{q}} \frac{1}{2 T_{o}}[\delta \theta * q-\delta \theta * \bar{q}] d \Gamma-\int_{\Gamma_{q}} \frac{1}{2 T_{o}}[\delta \theta(t) Q(0)-\delta \theta(t) \bar{Q}(0)] d \Gamma \\
& -\int_{\Gamma_{T}} \frac{1}{2 T_{o}}[\delta \theta * q] d \Gamma-\int_{\Gamma_{T}} \frac{1}{2 T_{o}}[\delta \theta(t) Q(0)] d \Gamma \\
& +\int_{\Gamma_{T}} \frac{1}{2 T_{o}}[\delta Q * T-\delta Q * \bar{T}] d \Gamma+\int_{\Gamma_{T}} \frac{1}{2 T_{o}}[\delta Q(t) \theta(0)-\delta Q(t) \bar{\theta}(0)] d \Gamma \\
& +\int_{\Gamma_{q}} \frac{1}{2 T_{o}}[\delta Q * T] d \Gamma+\int_{\Gamma_{q}} \frac{1}{2 T_{o}}[\delta Q(t) \theta(0)] d \Gamma=0
\end{aligned}
$$

The only differences from Eq. (24) are identified as second sound terms.

After assuming arbitrary variations, this leads to the following governing partial differential equations:

$$
\begin{array}{rr}
\frac{\rho_{o} c_{\varepsilon}}{T_{o}} \ddot{\theta}+\frac{1}{T_{o}} B_{i} \dot{H}_{i}=\frac{1}{T_{o}} \bar{\psi} & x \in \Omega, \tau \in(0, t) \\
-d_{i j} \tau_{o} \frac{1}{T_{o}} \ddot{H}_{j}-d_{i j} \frac{1}{T_{o}} \dot{H}_{j}-\frac{1}{T_{o}} B_{i} \dot{\theta}=0 & x \in \Omega, \tau \in(0, t)
\end{array}
$$

and initial conditions

$$
\frac{\rho_{o} c_{\varepsilon}}{T_{o}} \dot{\theta}(0)+\frac{1}{T_{o}} B_{i} H_{i}(0)=\frac{1}{T_{o}} \bar{\Psi}(0) \quad x \in \Omega
$$




$$
-d_{i j} \tau_{o} \frac{1}{T_{o}} \dot{H}_{j}(0)-d_{i j} \frac{1}{T_{o}} H_{j}(0)-\frac{1}{T_{o}} B_{i} \theta(0)=0 \quad x \in \Omega
$$

over the domain. The previous relations expressed in Eqs. (27)-(31) also apply without change for this heat diffusion problem with second sound. Thus, we have a new Principle of Stationary Mixed Convolved Action for a Linear Thermal Continuum undergoing non-classical heat diffusion involving the generalized Fourier law defined by Eq. (37b). This may be stated as follows: Of all the possible trajectories $\left\{\theta(\tau), H_{i}(\tau)\right\}$ of the system during the time interval $(0, t)$, the one that renders the action $I_{C_{G}}$ in Eq. (35) stationary, corresponds to the solution of the initial/boundary value problem of generalized heat diffusion with second sound.

\section{Time-space finite element methods for generalized heat diffusion}

In this section, we develop a true variational finite element approach for heat diffusion with second sound, based upon the principle of mixed convolved action. Unlike all previous work on dissipative continuum media, we use a finite element variational framework over both space and time.

Let us begin with the mixed convolved action $I_{C_{G}}$ defined in Eq. (35). After taking the variations, but before any integration by parts operations, we have

$$
\begin{aligned}
\delta I_{C_{G}} & =\int_{\Omega}\left[\delta \dot{\theta} * \frac{\rho_{o} c_{\epsilon}}{T_{o}} \dot{\theta}-\delta \dot{H}_{i} * d_{i j} \frac{\tau_{o}}{T_{o}} \dot{H}_{j}-\delta \breve{H}_{i} * d_{i j} \frac{1}{T_{o}} \breve{H}_{j}\right] d \Omega \\
& -\int_{\Omega}\left[\frac{1}{2}\left(\delta \breve{H}_{i} * \frac{1}{T_{o}} B_{i} \breve{\theta}-\delta \breve{\theta} * \frac{1}{T_{o}} B_{i} \breve{H}_{i}+\frac{1}{T_{o}} B_{i} \delta \breve{\theta} * \breve{H}_{i}-\frac{1}{T_{o}} B_{i} \delta \breve{H}_{i} * \breve{\theta}\right)\right] d \Omega \\
& -\int_{\Omega}\left[\delta \breve{\theta} * \frac{1}{T_{o}} \breve{\bar{\Psi}}\right] d \Omega+\int_{\Gamma_{q}} \frac{1}{2 T_{o}}[\delta \breve{\theta} * \breve{\bar{Q}}] d \Gamma-\int_{\Gamma_{T}} \frac{1}{2 T_{o}}[\delta \breve{Q} * \bar{\theta}] d \Gamma=0
\end{aligned}
$$

If Eq. (39) were used directly as the basis for the finite element representation, then $C^{0}$ continuity would be required for the fundamental impulsive variables $\theta$ and $H_{i}$ over both space and time. This is due to the appearance of first order spatial and temporal derivatives of each of these variables in Eq. (39).

Here, we choose to retain all of the temporal derivatives in Eq. (39) and employ linear shape functions for $\theta$ and $H_{i}$ in time to maintain the proper $C^{0}$ level of continuity. On the other hand, 
we use standard spatial integration by parts to eliminate all spatial derivatives of the heat vector $H_{i}$ and its variations in the weak form. As a result, the heat vector may be represented with piecewise constant values, which within the context of a finite element method translates to an element by element definition of the heat vector at each instant of time. In order to arrive at that formulation, we must perform spatial integration by parts on the second and fourth terms on the second line of Eq. (39). Of course, these operations also release boundary terms that must be considered within the finite element formulation. Our final weak form can be written as follows:

$$
\begin{aligned}
\delta I_{C_{G}} & =\int_{\Omega}\left[\delta \dot{\theta} * \frac{\rho_{o} c_{\epsilon}}{T_{o}} \dot{\theta}-\delta \dot{H}_{i} * d_{i j} \frac{\tau_{o}}{T_{o}} \dot{H}_{j}-\delta \breve{H}_{i} * d_{i j} \frac{1}{T_{o}} \breve{H}_{j}\right] d \Omega \\
& -\int_{\Omega}\left[\delta \breve{H}_{i} * \frac{1}{T_{o}} B_{i} \breve{\theta}+\frac{1}{T_{o}} B_{i} \delta \breve{\theta} * \breve{H}_{i}\right] d \Omega-\int_{\Omega}\left[\delta \breve{\theta} * \frac{1}{T_{o}} \breve{\bar{\Psi}}\right] d \Omega \\
& +\int_{\Gamma_{q}} \frac{1}{2 T_{o}}[\delta \breve{\theta} *(\breve{\bar{Q}}+\breve{Q})] d \Gamma-\int_{\Gamma_{T}} \frac{1}{2 T_{o}}[\delta \breve{Q} *(\breve{\bar{\theta}}-\breve{\theta})] d \Gamma=0
\end{aligned}
$$

Notice that this involves both classical first derivatives and fractional semi-derivatives in time, whereas the only spatial derivative operators included in Eq. (40) involve the gradients $\left(B_{i}\right)$ of the real temperature impulse semi-derivative $(\breve{\theta})$ and its variation $(\delta \breve{\theta})$.

Next, as an initial formulation, we restrict consideration to two-dimensional problems and introduce a simple finite element discretization over that space using standard three-node triangular elements. We start by performing integration over the domain $\Omega_{e}$ of an arbitrary threenode triangular element in the $x_{1}-x_{2}$ (or $x-y$ ) plane for each of the volumetric terms in Eq. (40). In performing this integration, we use a consistent representation of each term, meaning that we consider only the term with constant variation [46]. After the spatial discretization and integration, we may write

$$
\begin{gathered}
\int_{\Omega_{e}} \delta \dot{\theta} * \frac{\rho_{o} c_{\epsilon}}{T_{o}} \dot{\theta} d \Omega=\frac{1}{T_{o}} \delta \dot{\boldsymbol{\theta}}^{T} * \mathbf{M} \dot{\boldsymbol{\theta}} \\
\int_{\Omega_{e}} \delta \dot{H}_{i} * d_{i j} \frac{\tau_{o}}{T_{o}} \dot{H}_{j} d \Omega=\frac{1}{T_{o}} \delta \dot{\mathbf{H}}^{T} * \mathbf{A} \dot{\mathbf{H}} \\
\int_{\Omega_{e}} \delta \breve{H}_{i} * d_{i j} \frac{1}{T_{o}} \breve{H}_{j} d \Omega=\frac{1}{T_{o}} \delta \breve{\mathbf{H}}^{T} * \mathbf{D} \breve{\mathbf{H}}
\end{gathered}
$$




$$
\begin{gathered}
\int_{\Omega_{e}} \delta \breve{H}_{i} * \frac{1}{T_{o}} B_{i} \breve{\theta} d \Omega=\frac{1}{T_{o}} \delta \breve{\mathbf{H}}^{T} * \mathbf{B} \breve{\boldsymbol{\theta}} \\
\int_{\Omega_{e}} \frac{1}{T_{o}} B_{i} \delta \breve{\theta} * \breve{H}_{i} d \Omega=\frac{1}{T_{o}} \delta \breve{\boldsymbol{\theta}}^{T} \mathbf{B}^{T} * \breve{\mathbf{H}}
\end{gathered}
$$

For an isotropic solid, the matrices in Eqs. (41a-e) become

$$
\begin{gathered}
\mathbf{M}=\rho_{o} c_{\epsilon} A b \mathbf{I}_{3} / 3 \\
\mathbf{A}=\frac{\tau_{o}}{k} A b \mathbf{I}_{2} \\
\mathbf{D}=\frac{1}{k} A b \mathbf{I}_{2} \\
\mathbf{B}=\frac{b}{2}\left[\begin{array}{lll}
y_{2}-y_{3} & y_{3}-y_{1} & y_{1}-y_{2} \\
x_{3}-x_{2} & x_{1}-x_{3} & x_{2}-x_{1}
\end{array}\right]
\end{gathered}
$$

where $x_{q}$ and $y_{q}$ represent the coordinates of node $q$ of the triangular element, $A$ represents the area of the triangle, $b$ is the thickness, $k$ is the (isotropic) thermal conductivity and $\mathbf{I}_{p}$ is the $p \times p$ identity matrix.

Similarly, after spatial discretization, the body source contributions over an element can be written:

$$
\int_{\Omega_{e}} \delta \breve{\theta} * \frac{1}{T_{o}} \stackrel{\bar{\Psi}}{d} d \Omega=\frac{1}{T_{o}} \delta \breve{\boldsymbol{\theta}}^{T} * \check{\overline{\mathbf{\Psi}}}
$$

while the terms from the boundary conditions can be evaluated through integration over an element edge, thus yielding

$$
\begin{gathered}
\int_{\Gamma_{q}} \frac{1}{2 T_{o}}[\delta \breve{\theta} *(\breve{\bar{Q}}+\breve{Q})] d \Gamma=\frac{1}{2 T_{o}} \delta \breve{\boldsymbol{\theta}}^{T} *(\breve{\overline{\mathbf{Q}}}+\breve{\mathbf{Q}}) \\
\int_{\Gamma_{T}} \frac{1}{2 T_{o}}[\delta \breve{Q} *(\breve{\bar{\theta}}-\breve{\theta})] d \Gamma=\frac{1}{2 T_{o}} \delta \breve{\mathbf{Q}}^{T} *(\breve{\overline{\boldsymbol{\theta}}}-\breve{\boldsymbol{\theta}})
\end{gathered}
$$


With higher order spatial elements, there are some interesting ways to accommodate the influences defined in Equations $(44 a, b)$. In the present work with low order triangles, we simply equate the unknown variables to the known values (i.e., $\breve{\mathbf{Q}}=\check{\overline{\mathbf{Q}}}$ and $\breve{\boldsymbol{\theta}}=\breve{\overline{\boldsymbol{\theta}}}$ ) on edges associated with $\Gamma_{q}$ and $\Gamma_{T}$, such that the enforced heat fluxes have a contribution defined by

$$
\int_{\Gamma_{q}} \frac{1}{2 T_{o}}[\delta \breve{\theta} *(\breve{\bar{Q}}+\breve{Q})] d \Gamma=\frac{1}{T_{o}} \delta \breve{\boldsymbol{\theta}}^{T} * \overline{\mathbf{Q}}
$$

while the enforced temperature impulse integral has no explicit additional effect, because

$$
\int_{\Gamma_{T}} \frac{1}{2 T_{o}}[\delta \breve{Q} *(\breve{\bar{\theta}}-\breve{\theta})] d \Gamma=0
$$

After substituting Eqs. (41)-(43) and (45) into Eq. (40) and then factoring out the reference absolute temperature $T_{o}$ that appears in each term, the spatially discretized mixed weak form for an element can be written:

$$
\begin{array}{rl}
\delta \dot{\boldsymbol{\theta}}^{T} & * \mathbf{M} \dot{\boldsymbol{\theta}}-\delta \dot{\mathbf{H}}^{T} * \mathbf{A} \dot{\mathbf{H}}-\delta \breve{\mathbf{H}}^{T} * \mathbf{D} \breve{\mathbf{H}} \\
& -\delta \breve{\mathbf{H}}^{T} * \mathbf{B} \breve{\boldsymbol{\theta}}-\delta \breve{\boldsymbol{\theta}}^{T} \mathbf{B}^{T} * \breve{\mathbf{H}}-\delta \breve{\boldsymbol{\theta}}^{T} * \breve{\overline{\mathbf{\Psi}}}+\delta \breve{\boldsymbol{\theta}}^{T} * \breve{\overline{\mathbf{Q}}}=0
\end{array}
$$

The last term in Eq. (46) is understood to represent contributions from the known applied boundary heat fluxes, and is present only if such terms act on the edges of the element.

The weak form expressed either in spatially continuous or discrete form as Eq. (40) or Eq. (46), respectively, involves first derivatives of the temperature impulse ( $\theta$ or $\boldsymbol{\theta})$ and heat vector $\left(H_{i}\right.$ or $\left.\mathbf{H}\right)$. Consequently, these variables must be represented by $C^{0}$ continuous functions in time. In order to satisfy this minimum continuity requirement, for the temporal representation of the temperature impulse and heat vector field variables, we choose standard linear shape functions. Thus, over a time interval from $0 \leq t \leq \Delta t$, we write:

$$
\begin{aligned}
\boldsymbol{\theta}(t) & =\boldsymbol{\theta}_{0} N_{0}(t)+\boldsymbol{\theta}_{1} N_{1}(t) \\
\mathbf{H}(t) & =\mathbf{H}_{0} N_{0}(t)+\mathbf{H}_{1} N_{1}(t)
\end{aligned}
$$

in terms of the shape functions 


$$
N_{0}(t)=1-\frac{t}{\Delta t} ; \quad N_{1}(t)=\frac{t}{\Delta t}
$$

with similar variations for $\delta \boldsymbol{\theta}(t)$ and $\delta \mathbf{H}(t)$. Furthermore, we also assume

$$
\begin{gathered}
\overline{\mathbf{\Psi}}(t)=\overline{\boldsymbol{\Psi}}_{0} N_{0}(t)+\overline{\boldsymbol{\Psi}}_{1} N_{1}(t) \\
\overline{\mathbf{Q}}(t)=\overline{\mathbf{Q}}_{0} N_{0}(t)+\overline{\mathbf{Q}}_{1} N_{1}(t)
\end{gathered}
$$

although one may instead work with the given functions $\overline{\mathbf{\Psi}}(t)$ and $\overline{\mathbf{Q}}(t)$ directly.

After substituting Eqs. (47)-(49) into Eq. (46), performing the necessary convolution integrals in closed form, and collecting terms, we may write the following weak form:

$$
\begin{aligned}
& \left\{\begin{array}{llll}
\delta \boldsymbol{\theta}_{0}^{T} & \delta \boldsymbol{\theta}_{1}^{T} & \delta \mathbf{H}_{0}^{T} & \delta \mathbf{H}_{1}^{T}
\end{array}\right\} \\
& \left(\frac{1}{2 \Delta t}\left[\begin{array}{cccc}
2 \mathbf{M} & -2 \mathbf{M} & -\Delta t \mathbf{B}^{T} & \Delta t \mathbf{B}^{T} \\
-2 \mathbf{M} & 2 \mathbf{M} & \Delta t \mathbf{B}^{T} & \Delta t \mathbf{B}^{T} \\
-\Delta t \mathbf{B} & \Delta t \mathbf{B} & -2 \mathbf{A}+\Delta t \mathbf{D} & 2 \mathbf{A}-\Delta t \mathbf{D} \\
\Delta t \mathbf{B} & \Delta t \mathbf{B} & 2 \mathbf{A}-\Delta t \mathbf{D} & -2 \mathbf{A}-\Delta t \mathbf{D}
\end{array}\right]\left\{\begin{array}{c}
\boldsymbol{\theta}_{0} \\
\boldsymbol{\theta}_{1} \\
\mathbf{H}_{0} \\
\mathbf{H}_{1}
\end{array}\right\}-\left\{\begin{array}{c}
\frac{1}{2}\left(\boldsymbol{\Psi}_{1}-\boldsymbol{\Psi}_{0}\right) \\
\frac{1}{2}\left(\boldsymbol{\Psi}_{1}+\boldsymbol{\Psi}_{0}\right) \\
\mathbf{0} \\
\mathbf{0}
\end{array}\right\}\right)=0
\end{aligned}
$$

where now $\boldsymbol{\Psi}_{0}$ and $\boldsymbol{\Psi}_{1}$ are heat source impulses that collect all of the applied body source and surface heat flux contributions at times $t=0$ and $t=\Delta t$, respectively. Details for all of these temporal integrals, including those involving semi-derivatives, are provided in Reference [30].

For the first time step from $t=0$ to $t=\Delta t$, we assume that the field variables $\boldsymbol{\theta}_{0}$ and $\mathbf{H}_{0}$ are known and we seek to determine $\boldsymbol{\theta}_{1}$ and $\mathbf{H}_{1}$. For consistency, we must set the variations $\delta \boldsymbol{\theta}_{0}=\mathbf{0}$ and $\delta \mathbf{H}_{0}=\mathbf{0}$, while allowing $\delta \boldsymbol{\theta}_{1}$ and $\delta \mathbf{H}_{1}$ to remain arbitrary. As a result, after multiplying through by $4 / \Delta t$, Eq. (50) can be reduced to the following set of linear algebraic equations for each element:

$$
\frac{4}{(\Delta t)^{2}}\left[\begin{array}{cc}
\mathbf{M} & \frac{\Delta t}{2} \mathbf{B}^{T} \\
\frac{\Delta t}{2} \mathbf{B} & -\mathbf{A}_{1}
\end{array}\right]\left\{\begin{array}{c}
\boldsymbol{\theta}_{1} \\
\mathbf{H}_{1}
\end{array}\right\}=\frac{2}{\Delta t}\left\{\begin{array}{c}
\boldsymbol{\Psi}_{1}+\boldsymbol{\Psi}_{0} \\
\mathbf{0}
\end{array}\right\}+\frac{4}{(\Delta t)^{2}}\left[\begin{array}{cc}
\mathbf{M} & -\frac{\Delta t}{2} \mathbf{B}^{T} \\
-\frac{\Delta t}{2} \mathbf{B} & -\mathbf{A}_{0}
\end{array}\right]\left\{\begin{array}{c}
\boldsymbol{\theta}_{0} \\
\mathbf{H}_{0}
\end{array}\right\}
$$

where

$$
\mathbf{A}_{0}=\mathbf{A}-\frac{\Delta t}{2} \mathbf{D} ; \quad \mathbf{A}_{1}=\mathbf{A}+\frac{\Delta t}{2} \mathbf{D}
$$


Notice that the system matrix on the left-hand side of Eq. (51) is symmetric due to the symmetry of the $\mathbf{M}$ and $\mathbf{A}_{1}$ submatrices. However, rather than solving Eq. (51) in the present form, there is an opportunity to modify the solution sequence. In particular, because the heat vector is interpolated element-by-element as a $C^{-1}$ function, the $\mathbf{H}_{1}$ variables may be condensed out of the system at the element level. Solving the second set in Eq. (51) for $\mathbf{H}_{1}$ produces the following:

$$
\mathbf{H}_{1}=\mathbf{A}_{1}^{-1}\left(\frac{\Delta t}{2} \mathbf{B} \boldsymbol{\theta}_{1}+\frac{\Delta t}{2} \mathbf{B} \boldsymbol{\theta}_{0}+\mathbf{A}_{0} \mathbf{H}_{0}\right)
$$

After substituting Eq. (53) into the first set of Eq. (51), we may write the condensed relations for an element in the convenient form:

$$
\mathbf{K}_{1}^{e} \boldsymbol{\theta}_{1}=\boldsymbol{\psi}_{1}^{e}
$$

where

$$
\begin{gathered}
\mathbf{K}_{1}^{e}=\mathbf{B}^{T} \mathbf{A}_{1}^{-1} \mathbf{B}+\frac{4}{(\Delta t)^{2}} \mathbf{M} \\
\mathbf{K}_{0}^{e}=\mathbf{B}^{T} \mathbf{A}_{1}^{-1} \mathbf{B}-\frac{4}{(\Delta t)^{2}} \mathbf{M} \\
\mathbf{B}_{0}^{T}=\frac{1}{2} \mathbf{B}^{T}\left(\mathbf{I}_{2}+\mathbf{A}_{1}^{-1} \mathbf{A}_{0}\right) \\
\boldsymbol{\Psi}_{1}^{e}=\frac{2}{\Delta t}\left(\boldsymbol{\Psi}_{1}+\boldsymbol{\Psi}_{0}\right)-\mathbf{K}_{0}^{e} \boldsymbol{\theta}_{0}-\frac{4}{\Delta t} \mathbf{B}_{0}^{T} \mathbf{H}_{0}
\end{gathered}
$$

with the superscript ${ }^{e}$ as a reminder that all of these relations are written at the individual element level.

This can now be generalized for any step $n$ in which we move from a known solution at time $t_{n-1}$ to a solution at time $t_{n}$ with $\Delta t=t_{n}-t_{n-1}$. The resulting set of finite element equations, based on the principle of mixed convolved action for heat diffusion with second sound, can be written in the following form for each element:

$$
\mathbf{K}_{1}^{e} \boldsymbol{\theta}_{n}=\boldsymbol{\psi}_{n}^{e}
$$

where

$$
\boldsymbol{\Psi}_{n}^{e}=\frac{2}{\Delta t}\left(\boldsymbol{\Psi}_{n}+\boldsymbol{\Psi}_{n-1}\right)-\mathbf{K}_{0}^{e} \boldsymbol{\theta}_{n-1}-\frac{4}{\Delta t} \mathbf{B}_{0}^{T} \mathbf{H}_{n-1}
$$


Now let us consider the special case of classical heat diffusion in an isotropic solid, based upon the Fourier law of heat conduction. For this case, the relaxation time $\tau_{o}$ is effectively zero and $\mathbf{A}$ reduces to a null matrix. Consequently,

$$
\mathbf{A}_{1}=\frac{\Delta t}{2} \mathbf{D}=-\mathbf{A}_{0}
$$

and then

$$
\mathbf{K}_{1}^{e} \boldsymbol{\theta}_{n}=\boldsymbol{\psi}_{n}^{e}
$$

with

$$
\begin{gathered}
\mathbf{K}_{1}^{e}=\frac{2}{\Delta t} \mathbf{B}^{T} \mathbf{D}^{-1} \mathbf{B}+\frac{4}{(\Delta t)^{2}} \mathbf{M} \\
\mathbf{K}_{0}^{e}=\frac{2}{\Delta t} \mathbf{B}^{T} \mathbf{D}^{-1} \mathbf{B}-\frac{4}{(\Delta t)^{2}} \mathbf{M} \\
\mathbf{B}_{0}^{T}=\frac{1}{2} \mathbf{B}^{T}\left(\mathbf{I}_{2}-\mathbf{D}^{-1} \mathbf{D}\right)=\mathbf{0} \\
\boldsymbol{\Psi}_{n}^{e}=\frac{2}{\Delta t}\left(\boldsymbol{\Psi}_{n}+\boldsymbol{\Psi}_{n-1}\right)-\mathbf{K}_{0}^{e} \boldsymbol{\theta}_{n-1}
\end{gathered}
$$

Interestingly, because $\mathbf{B}_{0}$ reduces to a null matrix for classical heat diffusion, the right hand side of the discretized set of equations for this mixed convolved action formulation no longer depends explicitly on the previous values of the heat vector $\mathbf{H}_{n-1}$.

All of this has been developed at the element level. However, with the element by element elimination of the heat vector, the formulation looks quite similar to a standard temperature-based finite element method. Consequently, global system assembly follows the usual procedure and the final set of equations to be solved at each time step is as follows:

$$
\mathbf{K}_{1} \boldsymbol{\Theta}_{n}=\boldsymbol{\Psi}_{n}
$$

where $\boldsymbol{\Theta}_{n}$ collects all of the nodal temperature impulses at time step $n$.

Let us consider this a little further by multiplying Eq. (61) through by $\Delta t / 4$ and recognizing that $\mathbf{B}^{T} \mathbf{D}^{-1} \mathbf{B}$ represents the usual finite element conductivity matrix, which we will write as $\mathbf{C}$. The result is then

$$
\mathbf{M}\left(\frac{\boldsymbol{\theta}_{n}-\boldsymbol{\theta}_{n-1}}{\Delta t}\right)+\mathbf{C}\left(\frac{\boldsymbol{\theta}_{n}+\boldsymbol{\theta}_{n-1}}{2}\right)=\left(\frac{\overline{\boldsymbol{\Psi}}_{n}+\overline{\boldsymbol{\Psi}}_{n-1}}{2}\right)
$$


This is reminiscent of the familiar weighted residual approach for the heat equation, which can be written as

$$
\mathbf{M}\left(\frac{\mathbf{T}_{n}-\mathbf{T}_{n-1}}{\Delta t}\right)+\mathbf{C}\left(\beta \mathbf{T}_{n}+(1-\beta) \mathbf{T}_{n-1}\right)=\left(\beta \overline{\boldsymbol{\Psi}}_{n}+(1-\beta) \overline{\boldsymbol{\Psi}}_{n-1}\right)
$$

where $\mathbf{T}_{n}$ and $\overline{\boldsymbol{\psi}}_{n}$ are the nodal temperatures and applied heat sources, respectively, at time step $m$. For the Crank-Nicolson method [47], $\beta=1 / 2$ and Eq. (63) simplifies to the following:

$$
\mathbf{M}\left(\frac{\mathbf{T}_{n}-\mathbf{T}_{n-1}}{\Delta t}\right)+\mathbf{C}\left(\frac{\mathbf{T}_{n}+\mathbf{T}_{n-1}}{2}\right)=\left(\frac{\overline{\boldsymbol{\Psi}}_{n}+\overline{\boldsymbol{\Psi}}_{n-1}}{2}\right)
$$

which is identical to the variational mixed convolved action formulation, except that Eq. (62) is written in terms of impulsive quantities $\boldsymbol{\Theta}$ and $\overline{\mathbf{\Psi}}$, rather than $\mathbf{T}$ and $\overline{\boldsymbol{\psi}}$.

Thus, the simplest possible mixed convolved action time-space finite element method developed here has much in common with the well-known Crank-Nicolson method. Of course, the new mixed convolved action approach can be easily extended in many ways to formulate higher order methods, all founded on a rigorous variational framework.

\section{Computational Examples}

In this section, we consider two example problems to validate the mixed convolved action finite element formulation and to explore the response of solid media at small spatial and temporal scales, where the effect of finite thermal propagation speeds may become important.

\subsection{Unidirectional heat diffusion due to suddenly applied boundary heat flux}

First, we examine the problem of classical uniaxial heat diffusion in a uniform isotropic rectangular domain, as shown in Fig. 2. Notice that a heat flux is applied suddenly with unit magnitude all along the upper surface, such that $\bar{q}=H(t)$, with $H(t)$ representing the Heaviside step function. Meanwhile, the bottom surface is maintained at zero temperature, such that $\bar{T}=0$ on that edge. Non-dimensional geometric parameters and material properties are selected for the analysis, where $L=4, b=1, \rho_{o}=1, c_{\epsilon}=1$ and $k=1$, along with $\tau_{o}=0$ for this classical case. 


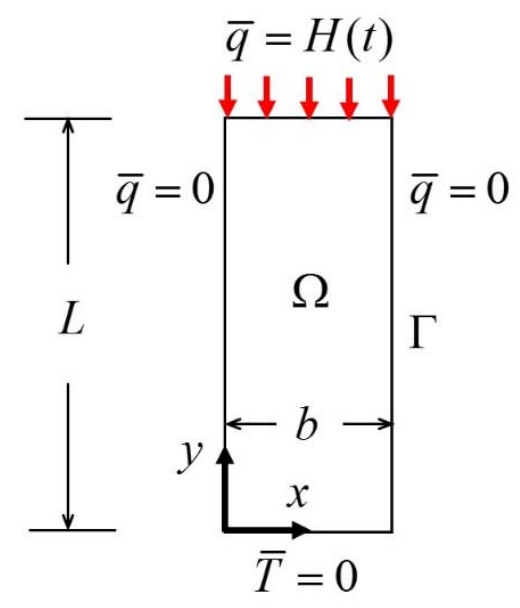

Fig. 2. Uniaxial heat diffusion in a rectangular domain.

Using separation of variables, the analytical solution can be found easily to have the following form:

$$
T(y, t)=\frac{y}{k}+\sum_{n=1}^{\infty}(-1)^{n} \frac{2}{k L c_{n}^{2}} \sin \left(c_{n} y\right) \exp \left(-c_{n}^{2} \kappa t\right)
$$

where $c_{n}=(2 n-1) \pi / 2 L$ and $\kappa=k / \rho_{o} c_{\epsilon}$ is the thermal diffusivity.

Fig. 3 provides the comparison of the temperatures at mid-height $(y=L / 2)$ and the top surface $(y=L)$ obtained from MCA and this analytical solution. The MCA results are displayed for three different levels of mesh refinement and time step duration $\Delta t$. There is some noticeable deviation of the MCA temperatures for the coarsest mesh and the longest time step, but this error diminishes significantly with increasing refinement of both the element sizes and time stepping. Clearly, the MCA approach converges to the analytical solution. Interestingly, this occurs at longer times, even for the coarsest mesh and time step for which the initial results deviate from the exact solutions near the beginning of the time interval. 


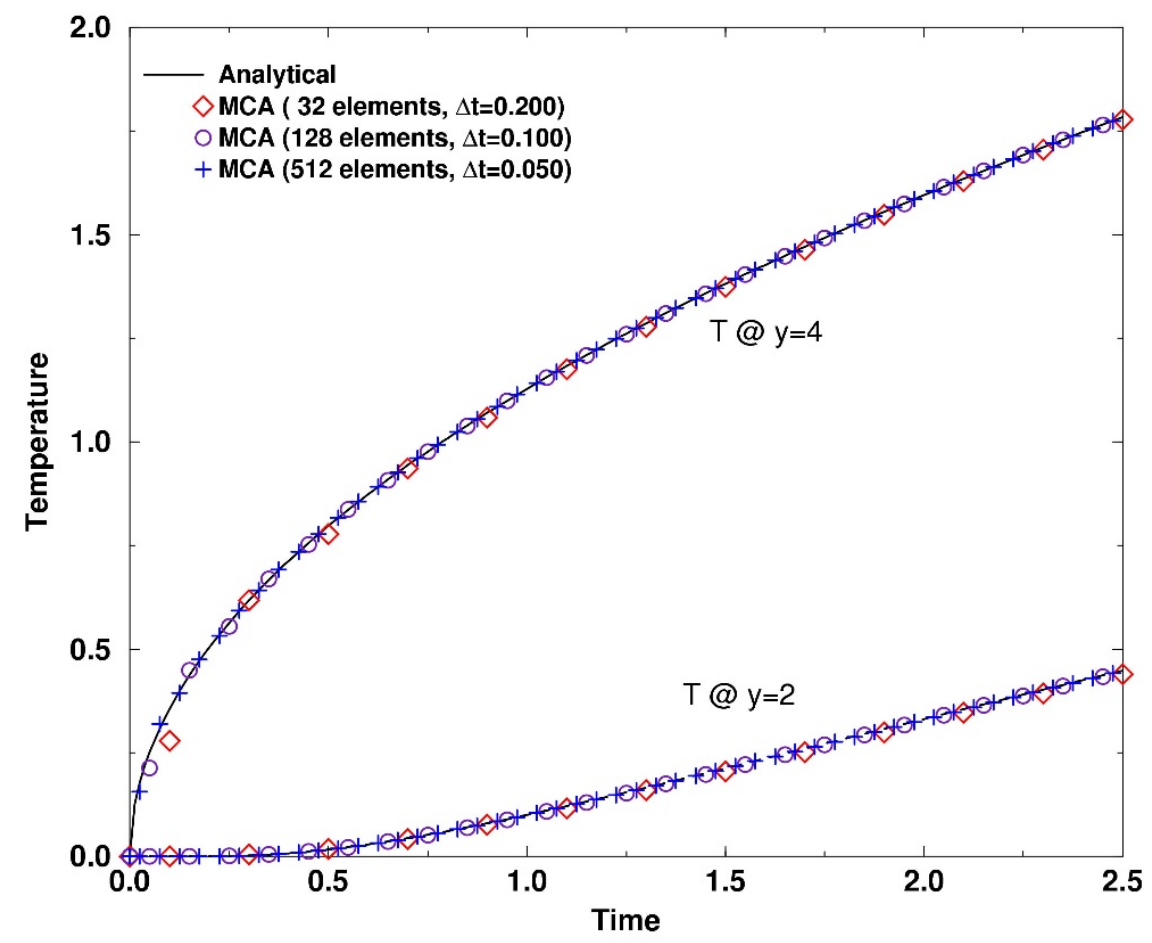

Fig. 3. Comparison of analytical and MCA temperatures for uniaxial heat diffusion.

\subsection{Pulse heating of square domain with thin insulating layer including second sound effects}

For the second problem, we examine the transient thermal response of a square domain with a localized pulse heat source applied to the top surface. Again, we use non-dimensional quantities and material parameters. As displayed in Fig. 4, the domain is $L$ by $L$, with $L=2$. The geometry is discretized into two sets of 800 uniform triangular elements to assure no biases are introduced. The heat source is spatially triangular, as shown in the problem schematic, and is a square pulse in time. The pulse has time duration $t_{d}=0.1$, for which the heat source is "on". This pulse is applied at the beginning of each period, where the time period is set to $t_{p}=1.0$, and then turned "off" for the remainder $t_{p}-t_{d}=0.9$ of each period. For all cases, we consider the amplitude of the pulse to be $q_{o}=1$. The pulse is applied centrally to the top surface and has width $b=0.2$. All other surfaces are considered to be insulated, as indicated in Fig. 4. 


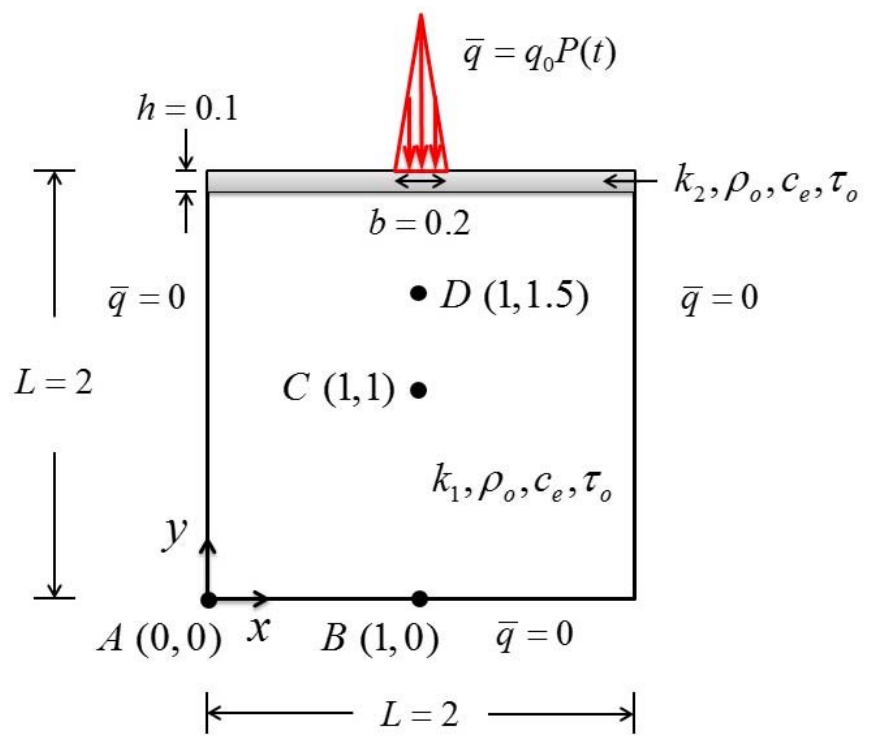

Fig. 4. Problem schematic for pulse heating of square domain.

For material properties, three different cases are analyzed. In all of the cases, we consider the following dimensionless properties: $\rho_{o}=1, c_{e}=1$ and for second sound relaxation time $\tau_{o}=0.1$. For the first case, we consider the upper layer having thickness $h=0.1$ to be the same material as the rest of the body, such that we have isotropic thermal conductivity $k_{1}=k_{2}=1$. For the second case, we have layer thermal conductivity $k_{2}=0.1$, and for the third case $k_{2}=0.01$.

Figs. 5-7 show the temporal evolution of temperature at four different points of interest (i.e., points $A, B, C$ and $D$ with the coordinates defined in Fig. 4). Fig. 5 corresponds to the first case with no insulating layer. The final data set on all plots corresponds to the temporal evolution of temperature at point $\mathrm{D}$ with no second sound effects (i.e., pure diffusion) for comparison. The significance of second sound effects is clear in this plot. Fig. 6 corresponds to the second case where we have an insulating layer with conductivity $k_{2}=0.1$. We notice that the effect is to shield the material in the sense that the temperature peaks decrease by nearly a factor of 2 compared to the uniform material case. More interestingly, we see that the temporal oscillations of temperature coming from second sound effects are noticeably smoothed. Fig. 7 then corresponds to the third case with a further reduced insulating layer conductivity $k_{2}=0.01$. Again this drastically shields the internal bulk material. Magnificently, however, we see that the temporal temperature profile begins to closely coincide with the pure diffusion case, such that the second sound effects are 
becoming increasingly negligible. This is the result of a filtering that occurs within the reduced thermal conductivity upper layer.

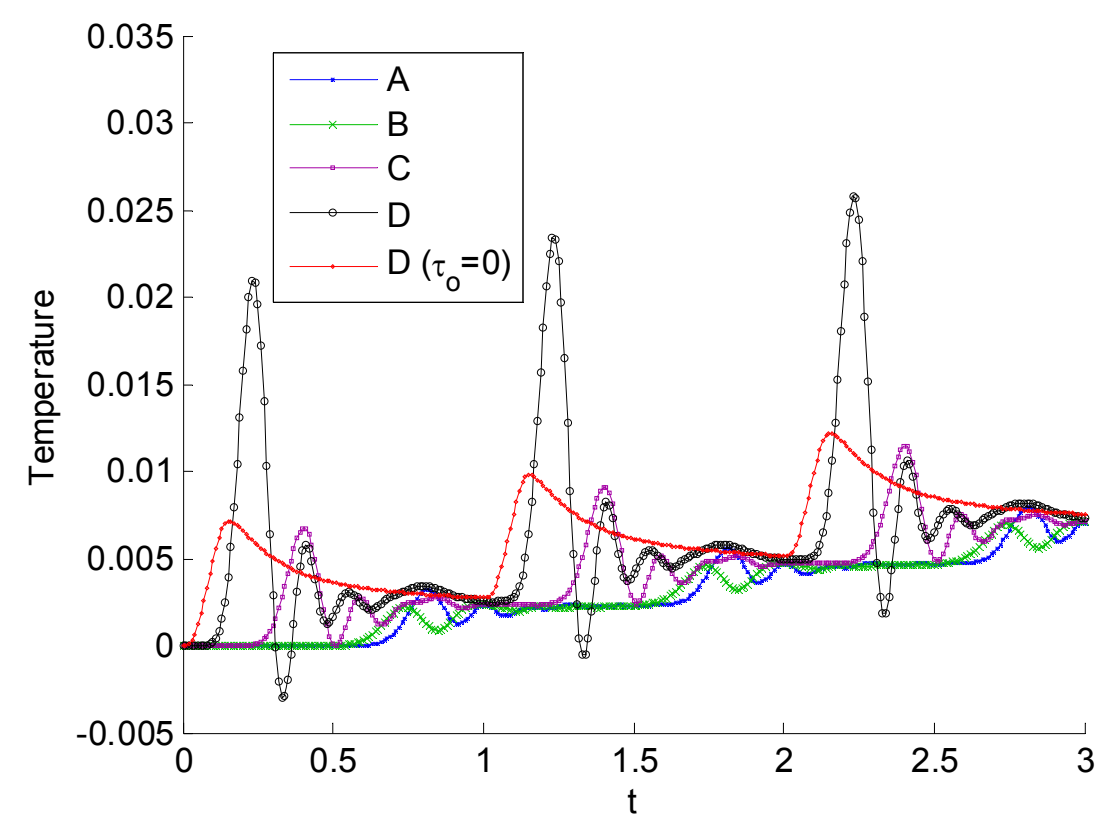

Fig. 5. Temperature versus time for points A-D; no layer.

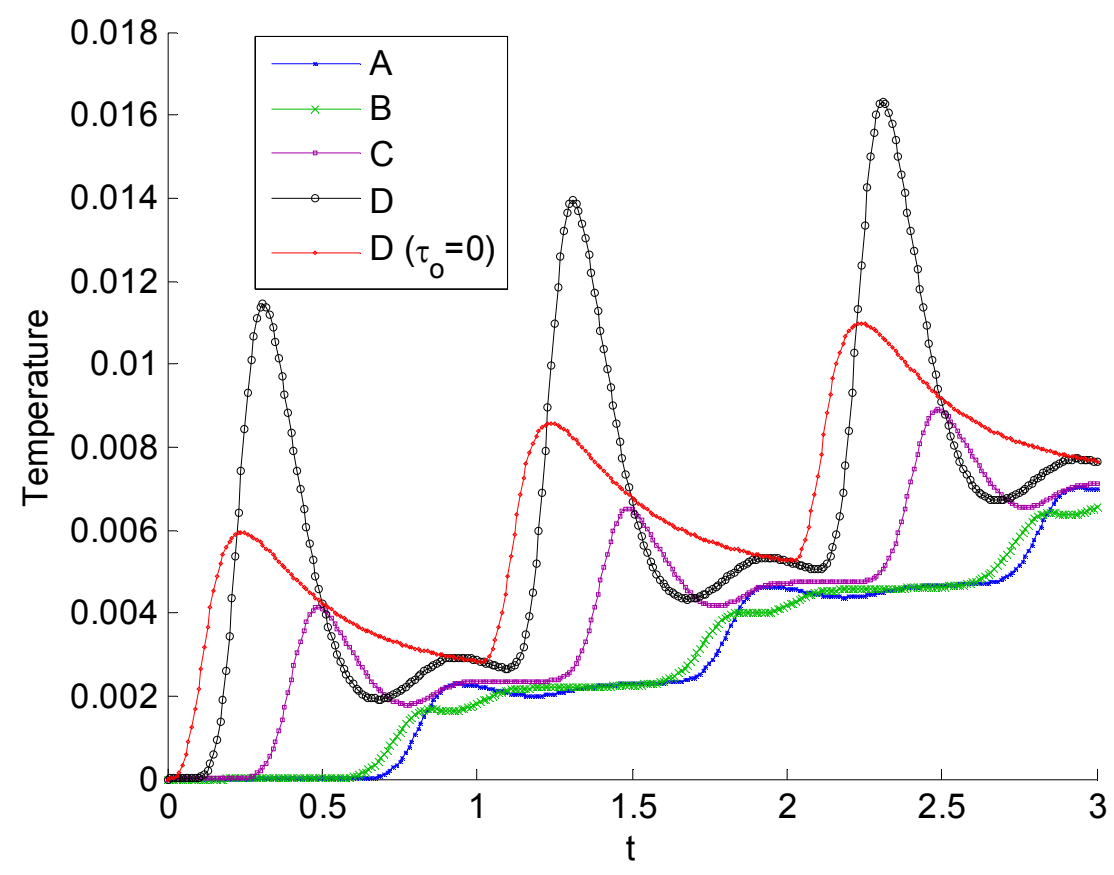

Fig. 6. Temperature versus time for points A-D; insulating layer with $k=0.1$. 


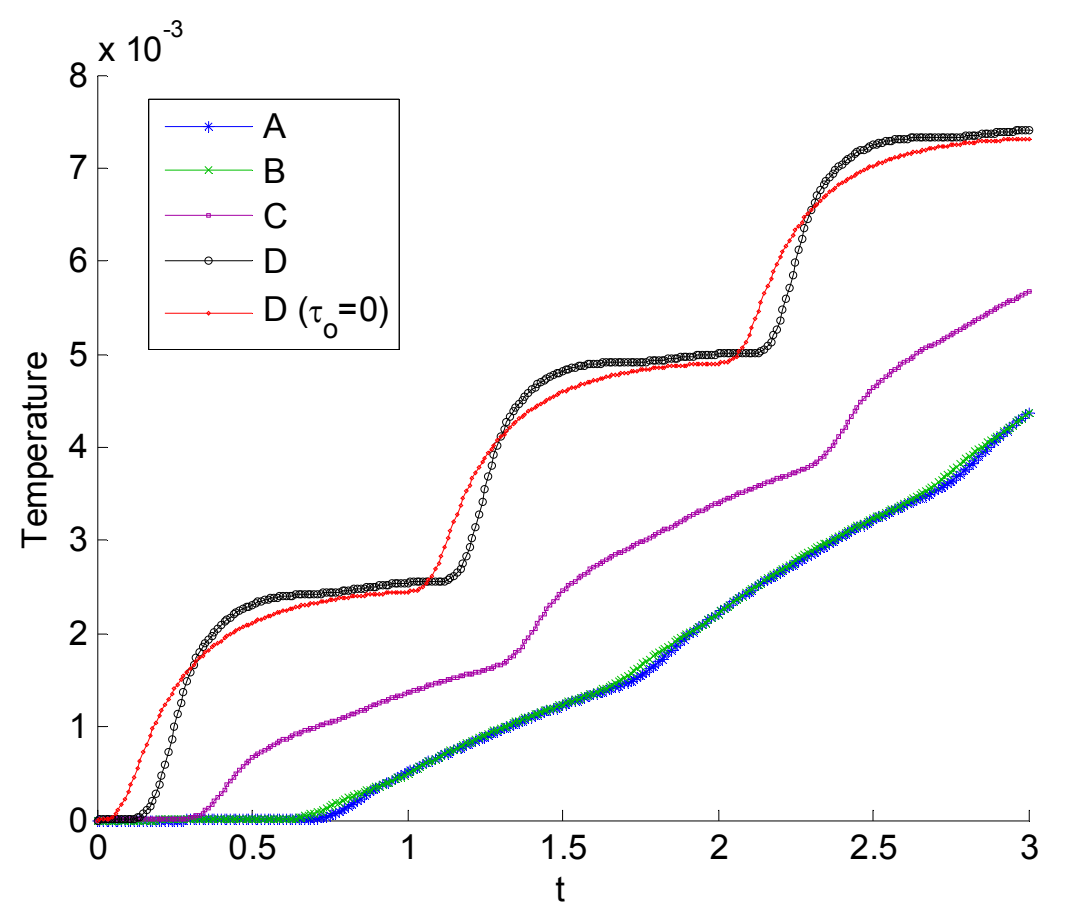

Fig. 7. Temperature versus time for points A-D; insulating layer with $k=0.01$.

\section{Conclusions}

Most modern-day computational approaches used to solve time-dependent problems of heat diffusion rely on a finite element representation over the spatial domain, along with a weighted residual or finite difference discretization to capture the temporal response. This space-time disparity can be attributed largely to the absence of a true variational framework for initial/boundary value problems, especially those involving dissipative processes. Historically, the main deficiency in variational methods, such as Hamilton's principle, for these problems has been the inability to capture the path-dependent nature of energy dissipation. The second main difficult has to do with improper handling of initial conditions, as opposed to boundary conditions. Both of these issues are associated with the inner product temporal representation in Hamilton's principle. This was identified long ago, especially by Gurtin and Tonti, who advocated for convolution-based operators instead.

To address this problem, a mixed convolved action has been developed here for purely dissipative heat diffusion, and then extended to include the possibility of second sound effects, all 
within the context of linear response. By introducing impulsive primary mixed variables and fractional derivatives, the present scalar action functional goes beyond previous formulations to encapsulate the complete initial/boundary value problem of heat diffusion. Remarkably, by enforcing stationarity of the mixed convolved action functional, the partial differential equations, initial conditions and boundary conditions are all recovered as the corresponding Euler-Lagrange equations. This variational framework is expressed as a new Principle of Stationary Mixed Convolved Action for Linear Thermal Continua.

In addition to the theoretical implications of this work, the mixed convolved action also may serve as a basis for novel computational methods. Here, we develop perhaps the simplest of those methods in the form of a space-time finite element for planar heat diffusion problems. This method employs three-node triangular elements, along with linear temporal shape functions. Interestingly, this formulation is shown to be closely aligned with the well-known Crank-Nicolson method for diffusion problems. The formulas are identical, but the present mixed convolved action uses impulse of temperature, rather than temperature, as the primary variables. Several examples are included to validate the mixed convolved action formulation and space-time finite element implementation.

With the thermal diffusion formulation written here in terms of impulse of temperature, we find one further conceptual advantage that should be mentioned. In this approach, the heat capacity contribution is associated with a thermal mass matrix, while the conductivity appears in the thermal damping matrix. Thus, in viewing a thermal problem in analogy with a structural or solid mechanics problem, we should think in terms of mass and damping matrices, rather than damping and stiffness matrices as is the usual current practice. The extensions to enforce finite speed of propagation of thermal disturbances also becomes much more direct with this approach, as was evident in the present work.

Finally, we should emphasize the major practical limitation of the present mixed convolved action formulation, which applies to linear problems only. The domain may involve heterogeneous, generally anisotropic media, but the classical convolution operator restricts consideration to linear systems. Thus, extensions are needed for more general nonlinear problems, for example, when thermal conductivity depends upon temperature. Several related variational approaches for such problems are currently under development. Furthermore, there is a need to 
focus on foundational aspects of the mixed convolved action formalism, especially to understand better the physical basis for this action and to explore its underlying geometric structure.

\section{References}

[1] W.R. Hamilton, On a general method in dynamics, Philosophical Transactions of the Royal Society of London 124 (1834) 247-308.

[2] W.R. Hamilton, Second essay on a general method in dynamics, Philosophical Transactions of the Royal Society of London 125 (1835) 95-144.

[3] J.W.S. Rayleigh, The Theory of Sound. I \& II, Dover Publications, New York, NY, 1877 (Second edition, reprint 1945).

[4] H. Goldstein, Classical Mechanics, Addison-Wesley Press, Reading, MA, 1980.

[5] L.D. Landau, E.M. Lifshitz, Mechanics, Pergamon Press, Oxford, UK, 1976.

[6] J.E. Marsden, T.S. Ratiu, Introduction to Mechanics and Symmetry: A Basic Exposition of Classical Mechanical Systems. Springer-Verlag, New York, NY, 1994.

[7] M.A. Biot, New methods in heat flow analysis with application to flight structures, Journal of the Aeronautical Sciences 24 (1957) 857-873.

[8] M.A. Biot, Variational Principles in Heat Transfer - A Unified Lagrangian Analysis of Dissipative Phenomena, Clarendon Press, Oxford, UK, 1970.

[9] H. Bateman, On dissipative systems and related variational principles, Physical Review 38 (1931) $815-$ 819.

[10] P.M. Morse, H. Feshbach, Methods of Theoretical Physics, McGraw-Hill, New York, NY, 1953.

[11] A.N. Kaufman, Dissipative Hamiltonian systems: A unifying principle, Physics Letters 100A (1984) 419-422.

[12] P.J. Morrison, Bracket formulation for irreversible classical fields, Physics Letters 100A (1984) 423427.

[13] M. Grmela, Bracket formulation of dissipative fluid mechanics equations, Physics Letters 102A (1984) $355-358$.

[14] A.N. Beris, B.J. Edwards, Thermodynamics of Flowing Systems with Internal Microstructure, Oxford University Press, New York, NY, 1994.

[15] M. Grmela, H.C. Ottinger, H.C., Dynamics and thermodynamics of complex fluids. I. Development of a general formalism, Physical Review E 56 (1997) 6620-6632.

[16] H.C. Ottinger, M. Grmela, Dynamics and thermodynamics of complex fluids. II. Illustrations of a general formalism, Physical Review E 56 (1997) 6633-6655.

[17] K.H. Anthony, A. Azirhi, Dislocation dynamics by means of Lagrange formalism of irreversible processes - complex fields and deformation processes, International Journal of Engineering Science 33 (1995) 2137-2148.

[18] B. Sievers, K.H. Anthony, Nonlocal Lagrange formalism in the thermodynamics of irreversible processes: Variational procedures for kinetic equations, Physica A 225 (1996) 89-128.

[19] K.H. Anthony, 2001. Hamilton's action principle and thermodynamics of irreversible processes - a unifying procedure for reversible and irreversible processes, Journal of Non-Newtonian Fluid Mechanics 96 (2001) 291-339. 
[20] F. Riewe, Nonconservative Lagrangian and Hamiltonian mechanics, Phys. Review E 53 (1996) 18901899.

[21] F. Riewe, Mechanics with fractional derivatives, Physical Review E 55 (1997) 3581-3592.

[22] J. Cresson, Fractional embedding of differential operators and Lagrangian systems, Journal of Mathematical Physics 48 (2007) 033504.

[23] M. Grmela, Contact geometry of mesoscopic thermodynamics and dynamics, Entropy 16 (2014) 16521686.

[24] M. Grmela, Geometry of multiscale nonequilibrium thermodynamics, Entropy 17 (2015) 5938-5964.

[25] M.E. Gurtin, Variational principles for linear initial-value problems, Quarterly of Applied Mathematics 22 (1964) 252-256.

[26] E. Tonti, On the variational formulation for linear initial value problems, Annali di Matematica Pura ed Applicata XCV (1973) 331-360.

[27] E. Tonti, A Systematic Approach to the Search for Variational Principles, Proceedings of the International Conference on Variational Principles in Engineering, Southampton Univ. Press (1973) $1.1-1.12$.

[28] G.F. Dargush, J. Kim, Mixed convolved action, Physical Review E 85 (2012) 066606.

[29] J. Kim, G.F. Dargush, Y-K. Ju, Extended framework of Hamilton's principle for continuum dynamics, International Journal of Solids and Structures 50 (2013) 3418-3429.

[30] G.F. Dargush, Mixed convolved action for classical and fractional-derivative dissipative dynamical systems, Physical Review E 86 (2012) 066606.

[31] G.F. Dargush, B.T. Darrall, J. Kim, G. Apostolakis, Mixed convolved action principles in linear continuum dynamics, Acta Mechanica, in press.

[32] M.E. Gurtin, Variational principles in the linear theory of viscoelasticity, Archive for Rational Mechanics and Analysis 13 (1963) 179-191.

[33] M.E. Gurtin, Variational principles for linear elastodynamics, Archive for Rational Mechanics and Analysis 16 (1964) 34-50.

[34] E. Tonti, Inverse problem: Its general solution, In: Differential Geometry, Calculus of Variations and Their Applications, Ed: G. M. Rassias, T. M. Rassias, Marcel Dekker, New York, NY, 1985.

[35] J.N. Reddy, Variational principles for linear coupled dynamic theory of thermoviscoelasticity, International Journal of Engineering Science, 14 (1976) 605-616.

[36] J.T. Oden, J.N. Reddy, Variational Methods in Theoretical Mechanics, Springer-Verlag, Berlin, 1983.

[37] K.B. Oldham, J. Spanier, The Fractional Calculus, Academic Press, New York, 1974.

[38] B. Ross, Fractional calculus. Mathematics Magazine 50 (1977) 115-122.

[39] S.G. Samko, A.A. Kilbas, O.I. Marichev, Fractional Integrals and Derivatives, Gordon and Breach Science Publishers, Switzerland, 1993.

[40] E.R. Love, L.C. Young, On fractional integration by parts, Proceedings of the London Mathematical Society, Series 244 (1938) 1-35.

[41] M.V. Sivaselvan, A.M. Reinhorn, Lagrangian approach to structural collapse simulation, Journal of Engineering Mechanics ASCE 132 (2006) 795-805.

[42] M.V. Sivaselvan, O. Lavan, G.F. Dargush, H. Kurino, Y. Hyodo, R. Fukuda, K. Sato, G. Apostolakis, A.M. Reinhorn, Numerical collapse simulation of large-scale structural systems using an optimizationbased algorithm, Earthquake Engineering and Structural Dynamics 38 (2009) 655-677.

[43] G. Apostolakis, G.F. Dargush, Mixed Lagrangian formulation for linear thermoelastic response of structures, Journal of Engineering Mechanics ASCE 138 (2012) 508-518.

[44] G. Apostolakis, G.F. Dargush, Mixed variational principles for dynamic response of thermoelastic and poroelastic continua, International Journal of Solids and Structures 50 (2013) 642-650. 
[45] G. Apostolakis, G.F. Dargush, Variational methods in irreversible thermoelasticity: Theoretical developments and minimum principles for the discrete form, Acta Mechanica 224 (2013) 2065-2088.

[46] G. Apostolakis, A Lagrangian Approach for Thermomechanics towards Damage and Deterioration of Structures, Ph.D dissertation, University at Buffalo, State University of New York, Buffalo, NY, 2010.

[47] J. Crank, P. Nicolson, A practical method for numerical evaluation of solutions of partial differential equations of the heat conduction type, Proceedings of the Cambridge Philosophical Society 43 (1947) $50-67$. 